\title{
Desempeño hidrológico y Propiedades Físico Mecánicas de Hormigones Porosos Elaborados con Áridos de la Provincia de Entre Ríos
}

\section{Hydrological Performance and Physico Mechanical Properties of Porous Concrete Developed with Aggregates from Entre Ríos Province}

\section{María Eugenia Garat}

Grupo de Investigación en Ingeniería Civil, Materiales y Ambiente (GIICMA) Universidad Tecnológica Nacional - Facultad Regional Concordia - Argentina eugarat@yahoo.com.ar

\section{Gustavo Roberto Larenze}

Grupo de Investigación en Ingeniería Civil, Materiales y Ambiente (GIICMA) Universidad Tecnológica Nacional - Facultad Regional Concordia - Argentina guslarenze@yahoo.com.ar

\section{Alberto José Palacio}

Grupo de Investigación en Ingeniería Civil, Materiales y Ambiente (GIICMA) Universidad Tecnológica Nacional - Facultad Regional Concordia - Argentina palacioalberto27@hotmail.com

\section{Jorge Daniel Sota}

Grupo de Investigación en Ingeniería Civil, Materiales y Ambiente (GIICMA) Universidad Tecnológica Nacional - Facultad Regional Concordia - Argentina jdsota@gmail.com 


\title{
RESUMEN
}

Los antecedentes vinculados al estudio de hormigones porosos en la República Argentina son escasos. Especialmente en nuestra región (Entre Ríos, cuenca del Río Uruguay) prácticamente no se dispone de información específica. Por ello, este trabajo se orientó a caracterizar las propiedades mecánicas e hidrológicas de hormigones porosos construidos con materiales locales. Se avanzó en el diseño de distintas mezclas, con porosidades variables hasta un máximo del 25\%. La resistencia a compresión se ubicó entre 9 y $39 \mathrm{Mpa}$, y la resistencia a tracción por flexión entre 1,4 y 2,4 Mpa, cuyos valores concuerdan con los reportados por la bibliografía. Se determinaron las tasas de infiltración de probetas limpias (Io en $\mathrm{mm} / \mathrm{h}$ ), las cuales están comprendidas entre $7000 \mathrm{~mm} / \mathrm{h}$ y $44.000 \mathrm{~mm} / \mathrm{h}$, y su variación en función del grado de colmatación, observando una drástica reducción hasta alcanzar la colmatación total (Ic en $\mathrm{mm} / \mathrm{h}$ ) con tasas comprendidas entre 20 y $45 \mathrm{~mm} / \mathrm{h}$.

Palabras clave: hormigón poroso, resistencia mecánica, infiltración.

\begin{abstract}
The background on the study of porous concrete in the Argentine Republic is limited. Especially in our region (Entre Ríos, Uruguay river basin) there is practically no specific information available. Therefore, this work was oriented to characterize the mechanical and hydrological properties of porous concrete constructed with local materials. Progress was made in the design of different mixtures, whose porosities are variable up to a maximum of $25 \%$. The compressive strength was between 9 and $39 \mathrm{Mpa}$, and the tensile strength by flex between 1.4 and $2.4 \mathrm{Mpa}$, whose values are consistent with those reported by the literature. Infiltration rates of clean specimens (Io in $\mathrm{mm} / \mathrm{h}$ ) were determined, which they are between $7000 \mathrm{~mm} / \mathrm{h}$ and 44,000 $\mathrm{mm} / \mathrm{h}$, and their variation according to the degree of clogging observing a drastic reduction until reaching the total clogging (Ic in $\mathrm{mm} / \mathrm{h}$ ) with rates between 20 and $45 \mathrm{~mm} / \mathrm{h}$.
\end{abstract}

Keywords: porous concrete, mechanical resistance, infiltration.

\section{Introducción}

El sistema de pavimento poroso constituye una variante de las técnicas de infiltración contempladas por los SUDS (Sistemas Urbanos de Drenaje Sostenible) o BMPs (Best Management Practices o Buenas Prácticas Ambientales), que promueve el movimiento del agua dentro de las capas situadas bajo la superficie del pavimento. Su principal característica es el almacenamiento temporal del escurrimiento superficial en una cámara o reservorio de grava permeable. De este modo, la reducción del escurrimiento superficial depende de la profundidad del reservorio subterráneo de grava y de la cantidad de agua que infiltra hacia el subsuelo (GIHHA, 2014).

Si bien a nivel internacional es muy amplia la experiencia vinculada a este tipo de hormigones, en la República Argentina los antecedentes disponibles son muy escasos, y 
especialmente en nuestra región (Provincia de Entre Ríos, Corredor del Río Uruguay) prácticamente no se dispone de información específica. Por ello, el Proyecto de Investigación "Análisis de la infiltración de agua de lluvia en hormigones porosos para el control en origen de inundaciones", desarrollado en la por el Grupo de Investigación en Ingeniería Civil y Medio Ambiente (GIICMA) de la Regional Concordia de la UTN planteó como principal objetivo la caracterización de las propiedades mecánicas e hidrológicas de hormigones porosos construidos con áridos de la Provincia de Entre Ríos.

\section{Desarrollo}

\section{Diseño de la mezcla de hormigón poroso}

La dosificación del hormigón poroso se obtuvo a partir de propuesta de diferentes dosificaciones de prueba (identificados como pastones numerados del 1 al 10), que fueron desarrolladas en tres etapas. En la primera etapa experimental (que comprende los pastones 1, 2 y 3) se utilizaron como base las proporciones de una mezcla patrón (Mezcla M10) propuesta por el Instituto del Cemento Portland Argentino (Hernández et al., 2008) variando el tipo de agregado grueso, la relación pasta/agregado y agua/cemento. La segunda etapa, que comprende los pastones 4, 5 y 6 se enfocó en modificaciones del origen del agregado, la relación agua-cemento, la incorporación de un aditivo y la forma de compactación. La dosificación se fijó en la tercera etapa (pastones 7, 8 y 9), donde sólo se varió la forma de compactación con el propósito de obtener distintos porcentajes de porosidad.

En todas las etapas de dosificación se empleó un Cemento Portland Compuesto (CPC 40 según IRAM 50000-2014). Se emplearon agregados gruesos tradicionales en la industria local del hormigón elaborado: canto rodado $10-20 \mathrm{~mm}$ del Río Uruguay, proveniente de la ciudad de Concordia y basalto triturado $10-20 \mathrm{~mm}$ con origen en la localidad de Puerto Yeruá, también en el departamento de Concordia. Con respecto a la cantidad de agregado grueso a utilizar, la bibliografía recomienda 1190 a $1480 \mathrm{~kg} / \mathrm{m}^{3}$ y una relación agregado grueso/material cementicio (AG/MC) que varía entre 4 a 4,5. En este estudio se trabajó con $1300 \mathrm{~kg} / \mathrm{m}^{3} \mathrm{de}$ árido grueso, adoptándose una relación $\mathrm{AG} / \mathrm{MC}$ variable entre 3,50 y 4.

Se utilizó un pequeño porcentaje de arena gruesa de cantera proveniente de la ciudad de Concordia para minimizar el efecto de microfisuración por retracción de la pasta y aumentar la trabajabilidad de la mezcla (Peso específico: 2,63 kg/m3, Módulo de finura: 2,59, Absorción 0,6\%). La bibliografía recomienda bajos contenidos de agregado fino (relaciones agregado fino con respecto al agregado grueso (AF/AG) comprendidas entre 5 y 30\%), cuidando que no se reduzca la permeabilidad del hormigón. En este caso se utilizó un 5\% de fino respecto al agregado grueso, buscando que la adherencia del mortero con las partículas de agregado sea la suficiente para evitar segregación y resistencias muy bajas. La distribución granulométrica de los agregados gruesos y del árido fino, así como las curvas límites según Norma IRAM 1627 se presentan en la Figura 1 y en la Figura 2 respectivamente.

Se empleó un aditivo reductor de agua de alto rango superplastificante, DARACEM ${ }^{\mathrm{TM}} 100$ HV, (GCP Applied Technologies. Densidad: 1,20 kg/lt. Dosis: 1,0\% del peso del cemento). La bibliografía consultada recomienda utilizar relaciones agua/cemento (a/c) comprendidas entre 0,27 y 0,30 en el caso de utilizar aditivos, y entre 0,34 a 0,40 si no se utilizan aditivos. En este trabajo se empleó una relación a/c de 0,28. 


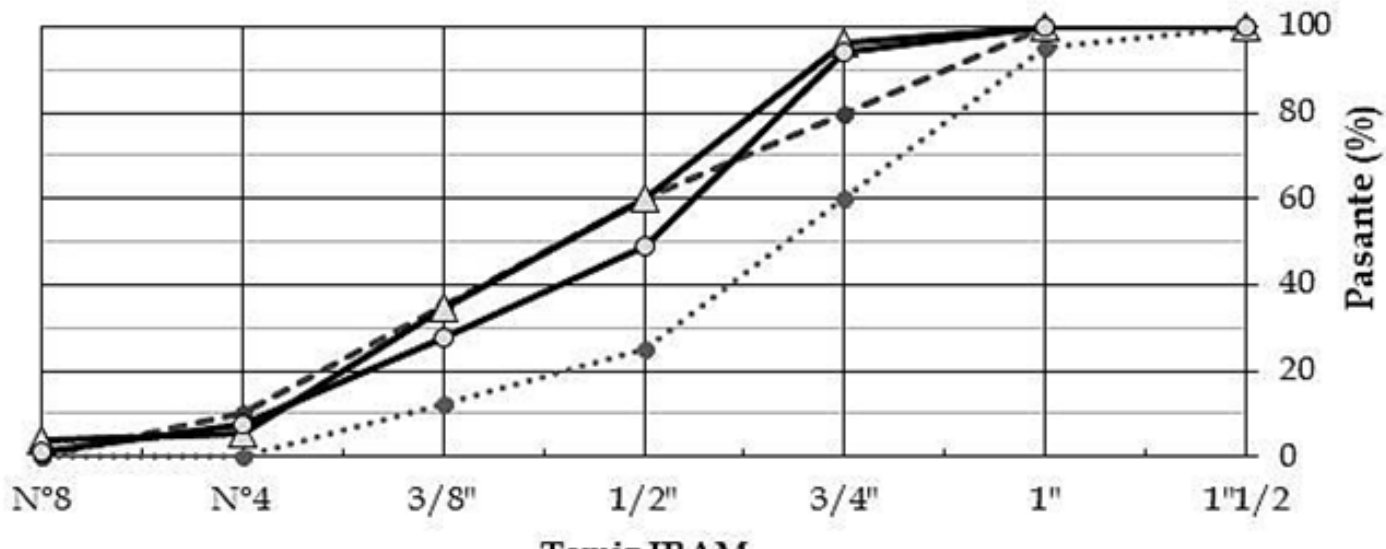

Tamiz IRAM

… Mínimo $-\infty-$ Máximo $\rightarrow$ BASALTO $\longrightarrow$ CANTO RODADO

Figura 1.- Curvas granulométricas de Agregado Basáltico y Canto Rodado. Según Norma IRAM 1627.

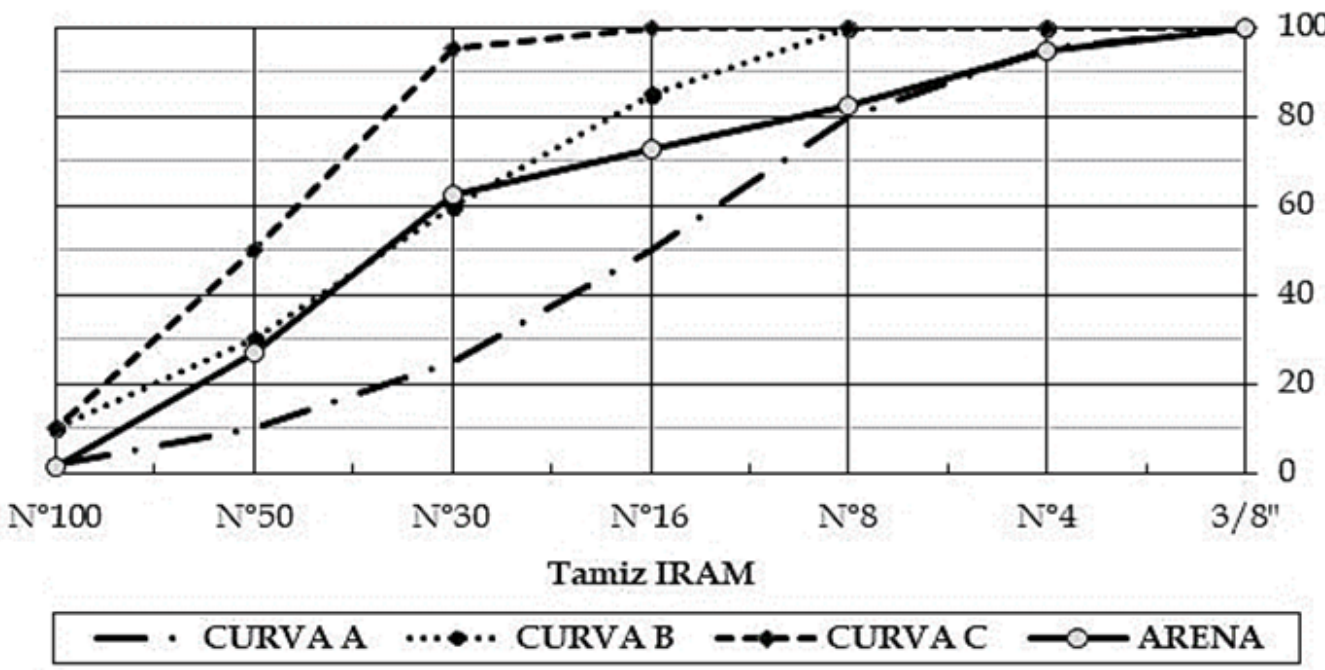

Figura 2.- Curva granulométrica de Agregado Fino.

Las dosificaciones correspondientes a la primera etapa experimental, que comprende los pastones 1,2 y 3, tomaron como referencia las proporciones de una mezcla patrón (Mezcla M10) propuesta por el Instituto del Cemento Portland Argentino (Hernández et al., 2008) que se adaptó a los materiales locales, variando el tipo de agregado grueso, la relación pasta/ agregado y agua/cemento. Esta primera etapa se orientó a obtener una mezcla trabajable, que cumpliera los requerimientos de resistencia en estado endurecido.

En el pastón 1 se empleó canto rodado, relación AF/AG del 5\% y una cantidad de cemento de $390 \mathrm{~kg} / \mathrm{m}^{3}$, con relaciones a/c de 0,22. A partir de los resultados obtenidos en el primer pastón, se modificó la dosificación inicial reemplazando el agregado grueso de la 
dosificación inicial por basalto triturado en el pastón 2, aumentando la relación a/c a 0,27 y manteniendo el resto de los parámetros constantes. Posteriormente, considerando los resultados anteriores, para el pastón 3 se empleó un aditivo superplastificante para mejorar las propiedades del hormigón en estado fresco (proporción 1\% del peso del cemento). La relación a/c utilizada fue de 0,25.

Se moldearon 6 probetas cilíndricas ( $15 \mathrm{~cm}$ de diámetro y $30 \mathrm{~cm}$ de altura) y 2 probetas prismáticas (vigas de $15 \times 15 \times 55 \mathrm{~cm}$ ) por pastón, para determinar las resistencias a compresión y flexión respectivamente.

La segunda etapa experimental de mezclas (pastones 4, 5 y 6) se orientó a incrementar la resistencia a compresión y ajustar la porosidad del hormigón. Se aumentó el contenido unitario de cemento a $420 \mathrm{~kg} / \mathrm{m}^{3}$, fijando la relación a/c en 0,26 y la relación agregado grueso/ cemento. Para el pastón 4 se empleó canto rodado y para los pastones 5 y 6 se empleó basalto triturado. Se elaboraron pastones de 50 litros en los que, además de evaluar su resistencia a compresión, se moldearon probetas prismáticas de 49x49x10 cm con el fin de evaluar las propiedades drenantes en el simulador de lluvia. Además, en cada uno de los pastones se determinó densidad y porosidad de las probetas moldeadas.

A partir de los resultados anteriores se plantearon nuevas dosificaciones (pastones 7, 8 y 9) que determinaron la tercera etapa experimental, donde se varió la porosidad del hormigón modificando el grado de compactación. Se mantuvo la dosificación 6, elaborada con basalto triturado y las proporciones de los agregados. Se fijó la relación a/c en 0,28. En todos los pastones se utilizó el aditivo superplastificante DARACEM 100. Se elaboraron pastones de 100 litros en los que se moldearon series de tres probetas prismáticas de 49x49x10 cm con el fin de realizar los ensayos de permeabilidad y ensayos con simulador de lluvia. Para los ensayos hidrológicos, los pastones 7, 8 y 9 se designaron con las letras A, B y C respectivamente.

Además se diseñó una última mezcla denominada pastón 10, de iguales características al 9 para elaborar el hormigón del pavimento a emplear en la prueba piloto. En este caso lo más significativo es que se varió el agregado grueso, empleándose el que poseía la planta elaboradora de hormigón en el obrador. Este también consistió en un basalto triturado 10-20, pero considerando que poseía un mayor porcentaje de fino, no se incorporó el 5\% de arena gruesa como agregado fino.

En la Tabla 1 se muestran las dosificaciones realizadas en cada etapa experimental.

\begin{tabular}{|c|c|c|c|c|c|c|c|c|c|c|}
\hline $\begin{array}{c}\text { Materiales } \\
{[\mathrm{kg}]}\end{array}$ & $\begin{array}{c}\text { Pastón } \\
1\end{array}$ & $\begin{array}{c}\text { Pastón } \\
2\end{array}$ & $\begin{array}{c}\text { Pastón } \\
3\end{array}$ & $\begin{array}{c}\text { Pastón } \\
4\end{array}$ & $\begin{array}{c}\text { Pastón } \\
5\end{array}$ & $\begin{array}{c}\text { Pastón } \\
6\end{array}$ & $\begin{array}{c}\text { Pastón } \\
7 \text { (A) }\end{array}$ & $\begin{array}{c}\text { Pastón } \\
8(\mathrm{~B})\end{array}$ & $\begin{array}{c}\text { Pastón } \\
\mathbf{9} \text { (C) }\end{array}$ & $\begin{array}{c}\text { Pastón } \\
\mathbf{1 0}\end{array}$ \\
\hline Agua & 87 & 105 & 119 & 105 & 117 & 115 & 121 & 124 & 123 & 110 \\
\hline Cemento & 390 & 390 & 390 & 420 & 420 & 420 & 420 & 420 & 420 & 420 \\
\hline $\begin{array}{c}\text { Aditivo } \\
\text { Daracem 100 }\end{array}$ & -- & -- & 4,21 & 4,20 & 4,20 & 4,20 & 4,20 & 4,20 & 4,20 & 4,20 \\
\hline $\begin{array}{c}\text { Canto Rodado } \\
\text { 1-2 }\end{array}$ & 1395 & -- & -- & 1388 & -- & -- & -- & -- & -- & -- \\
\hline Basalto 1-2 & -- & 1567 & 1552 & -- & 1371 & 1374 & 1390 & 1374 & 1366 & 1460 \\
\hline Arena Gruesa & 74 & 75 & 72 & 73 & 72 & 73 & 72 & 72 & 73 & -- \\
\hline
\end{tabular}

Tabla 1.- Dosificaciones para $1 \mathrm{~m} 3$ de los pastones realizados. 


\section{Propiedades del hormigón poroso}

\section{Propiedades en estado fresco}

Las propiedades a evaluar en estado fresco se relacionan principalmente con la trabajabilidad (afectada por la escasa o nula presencia de agregado fino y por la cantidad de agua) y la consistencia de la mezcla. Para cuantificarlas se realizaron los siguientes ensayos: Método de Hand-Squeeze, Asentamiento en el Tronco de cono de Abrams (IRAM 1536), Peso unitario (ASTM C1688), cuyo valor oscila entre 1600 y $2000 \mathrm{~kg} / \mathrm{m} 3$ según la bibliografía consultada.

\section{Propiedades en estado endurecido}

\section{Resistencia a compresión y a la tracción por flexión.}

Los ensayos se realizaron según Norma IRAM 1546, con una prensa electrohidráulica con una capacidad de 200 toneladas, empleando cabezales de madera laminada en superficies superior e inferior durante cada ensayo (Figura 3). Para la elaboración de las probetas, al igual que en la mezcla patrón, se empleó una compactación manual mediante el equipo Proctor Normal (T99) utilizado en el ensayo de compactación de suelos (Figura 4). Los moldes se llenaron en 3 capas de 18 golpes cada una (Hernández et al., 2008). Esta energía debe ser ajustada con la finalidad de lograr presiones similares a las de campo $\left(4 \mathrm{a} 7 \mathrm{t} / \mathrm{m}^{2}\right)$.

La resistencia a tracción por flexión varía generalmente entre 1,0 y 3,8 MPa. Su determinación puede estar sujeta a importante variabilidad, por lo que también es habitual medir la resistencia a compresión y usar relaciones empíricas para estimar su valor. Los ensayos de resistencia a tracción por flexión se realizan a la edad de 28 días según Norma IRAM 1547. En cuanto al modo de compactación de la probeta prismática, se dificulta aplicar en laboratorio la compactación de campo utilizada para pavimentos. En consecuencia, se decidió por compactar las probetas por impacto aplicando también el pisón del ensayo Proctor Estándar, mediante 2 capas de 18 golpes distribuidos en cada una. En la Figura 5 y la Figura 6 se puede observar la viga prismática empleada para el ensayo y el ensayo a flexión en tres puntos en ejecución.

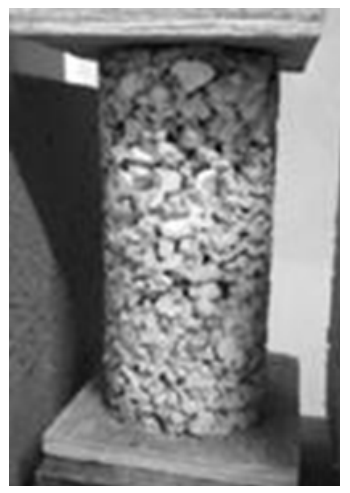

Figura 3.- Ensayo a compresión. 


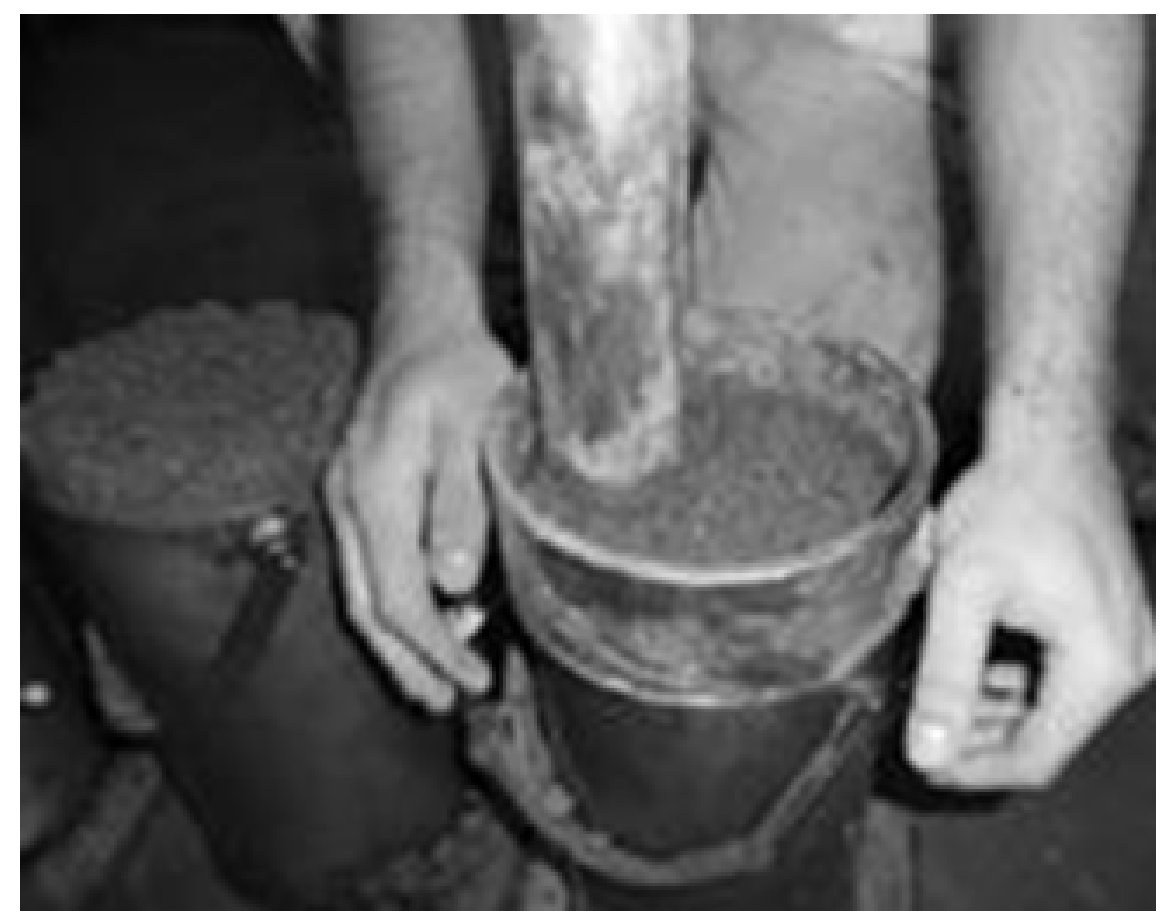

Figura 4.- Compactación de probetas cilíndricas.

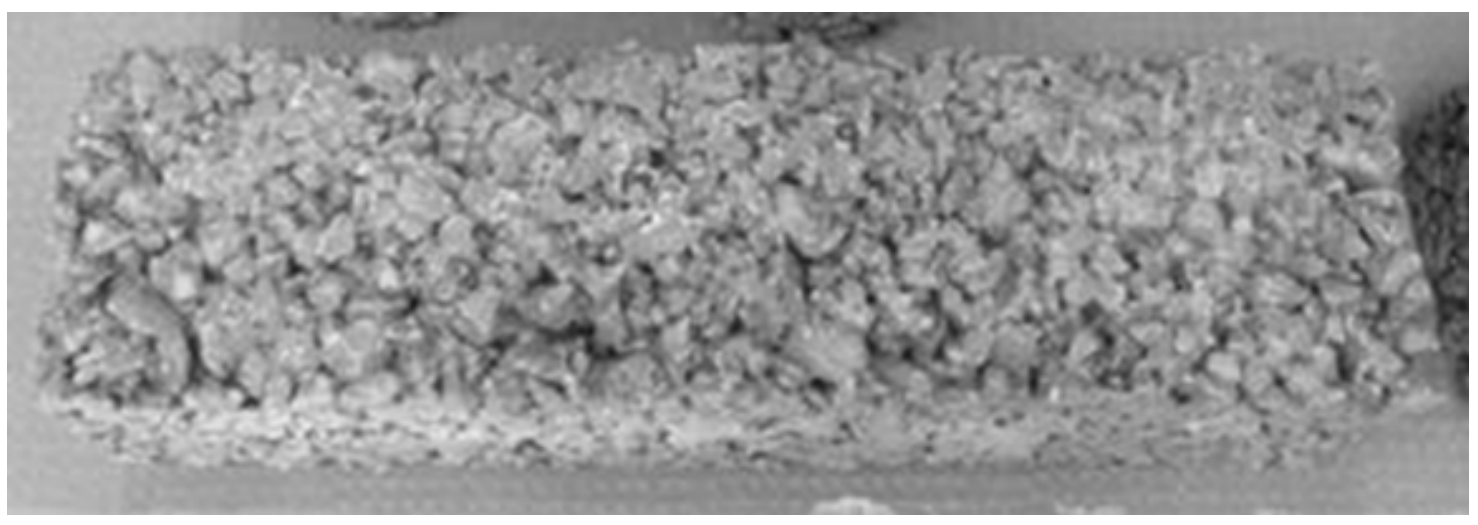

Figura 5.- Viga prismática de 15×15×55cm. 


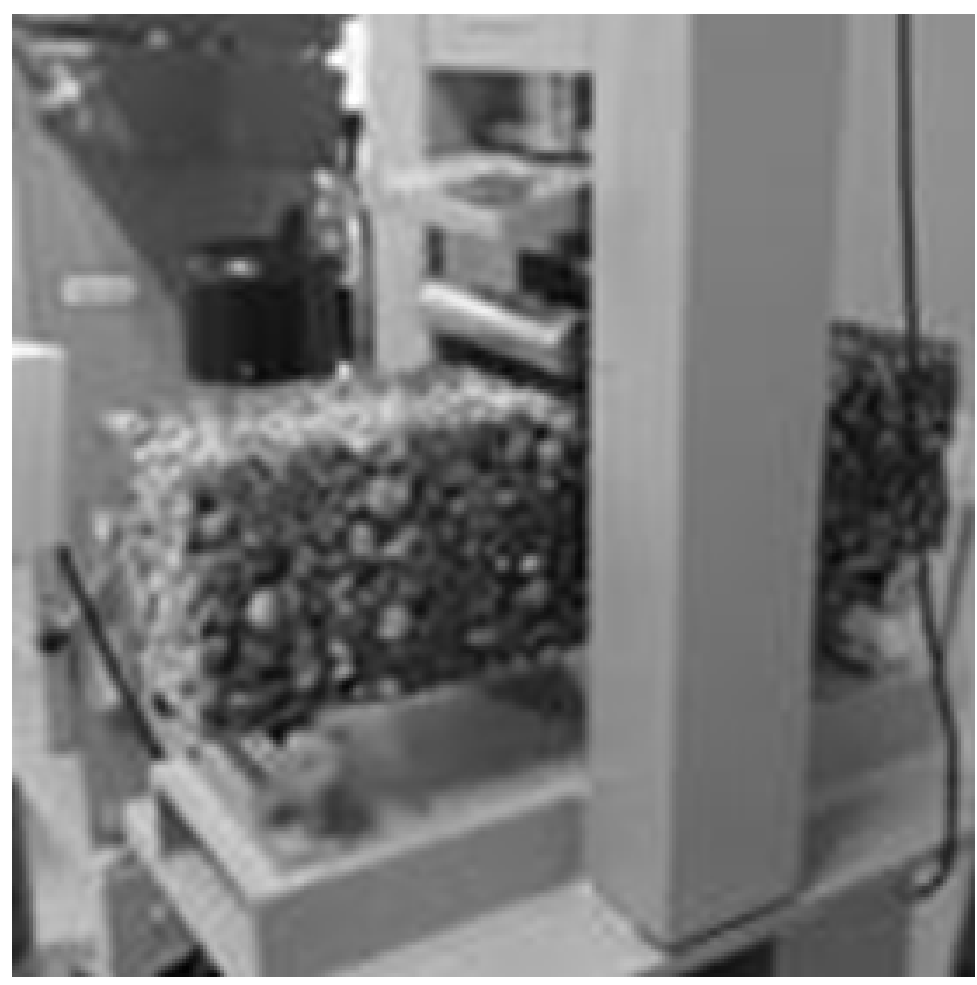

Figura 6.- Ensayo a flexión.

\section{Porosidad}

La porosidad es una de las características más importantes de este material. De acuerdo a la bibliografía especializada, para que el hormigón sea considerado poroso debe tener como mínimo un $15 \%$ de huecos, pero se recomienda que este contenido no supere el $25 \%$ debido a la poca estabilidad de la mezcla. Cuando el porcentaje de vacíos se ubica en el rango mencionado se obtienen mezclas con adecuada resistencia y permeabilidad.

Para compactar las probetas se empleó un rodillo de $120 \mathrm{~mm}$ de diámetro, $700 \mathrm{~mm}$ de ancho y $28 \mathrm{~kg}$ de peso (Figura 7), que proporciona presiones similares a las utilizadas en el terreno $(0,08 \mathrm{MPa})$. Para lograr los distintos valores de porosidad se determinó una relación empírica entre el peso de hormigón fresco colocado en el molde y el volumen de vacíos obtenido una vez realizada la compactación empleando el rodillo. Para determinar el porcentaje de vacíos, se empleó un método basado en el Principio de Arquímedes mediante el método de la balanza hidrostática. Mediante este ensayo, se obtiene el volumen de la estructura del hormigón poroso y el volumen de vacíos. Finalmente la porosidad del hormigón se determina calculando la diferencia de peso entre una muestra seca y sumergida 


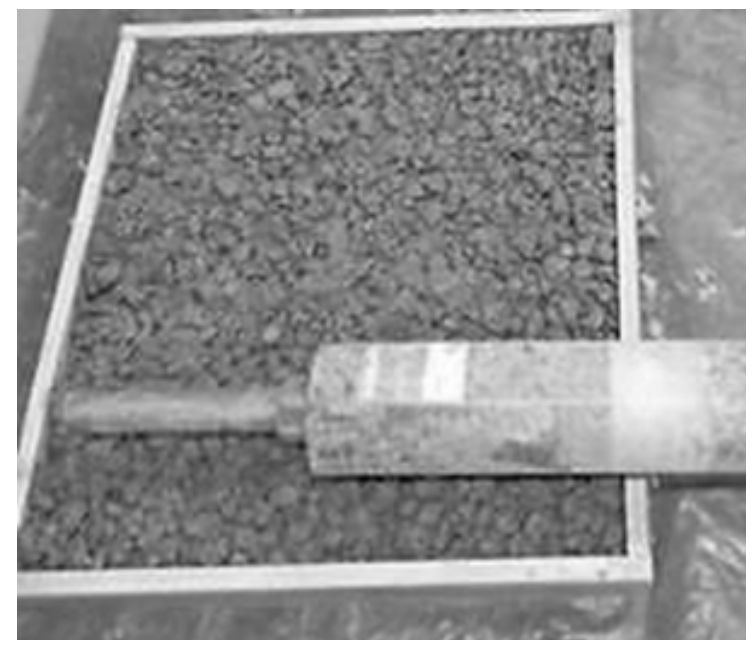

Figura 7.- Losa confeccionada mediante molde de madera y rodillo de compactación.

\section{Permeabilidad}

La permeabilidad depende de las propiedades de los materiales, dosificación de la mezcla y los métodos de colocación y compactación. Según la bibliografía analizada, la permeabilidad del hormigón poroso es muy elevada, y puede variar entre 0,2 a 5,0 cm/seg $(7200$ a $180.000 \mathrm{~mm} / \mathrm{h}$ ), es decir varios órdenes de magnitud superior a la intensidad de lluvia de las tormentas.

Las tasas de infiltración de muestras limpias o con contenidos de arena relativamente bajos se cuantificaron a partir del ensayo de permeabilidad ASTM C1701: "Standard Test Method for Infiltration Rate of In Place Pervious Concrete" (ASTM, 2009) que consiste en verter un volumen conocido de agua en un anillo de dimensiones normalizadas (que se sella sobre la superficie del pavimento permeable) y registrar el tiempo necesario para mantener un nivel de referencia constante de agua en su interior. El anillo es abierto en ambos extremos, debe tener un diámetro de $300 \pm 10 \mathrm{~mm}$ con una altura mínima de $50 \mathrm{~mm}$. En su interior se marcan dos líneas a distancias de 10 y $15 \mathrm{~mm}$ desde el fondo del anillo.

El ensayo se realiza en dos etapas: la primera consiste en una etapa de prehumedecimiento, que se utiliza para definir el volumen de agua que se utilizará en el ensayo de infiltración propiamente dicho. La segunda es el ensayo de infiltración propiamente dicho, que debe realizarse dentro de los dos minutos posteriores al prehumedecimiento. Se registra el tiempo requerido para que el agua infiltre en la superficie del pavimento, mientras se vierte a la velocidad necesaria para mantener el nivel entre las dos líneas mencionadas. La tasa de infiltración se calcula según Ecuación (1).

$$
i=\frac{K M}{D^{2} t}
$$


Siendo:

$\mathrm{i}=$ Tasa de infiltración $(\mathrm{mm} / \mathrm{h})$

$\mathrm{M}=$ Masa de agua $(\mathrm{kg})$

$\mathrm{D}=$ Diámetro interior del anillo de infiltración $(\mathrm{mm})$

$\mathrm{t}=$ Tiempo (segundos)

$\mathrm{K}=$ factor de conversión de unidades 4.583.666.000 en el Sistema Internacional $\left(\mathrm{mm}^{3} \mathrm{x} \mathrm{s}\right) /$ $(\mathrm{kg} / \mathrm{h})$

En la Figura 8 se presenta la ubicación del anillo de infiltración sobre las probetas ensayadas. En la Figura a) puede observarse que en la parte inferior del dispositivo de ensayo se ubicó una balanza para registrar la evolución temporal de la masa de agua infiltrada.

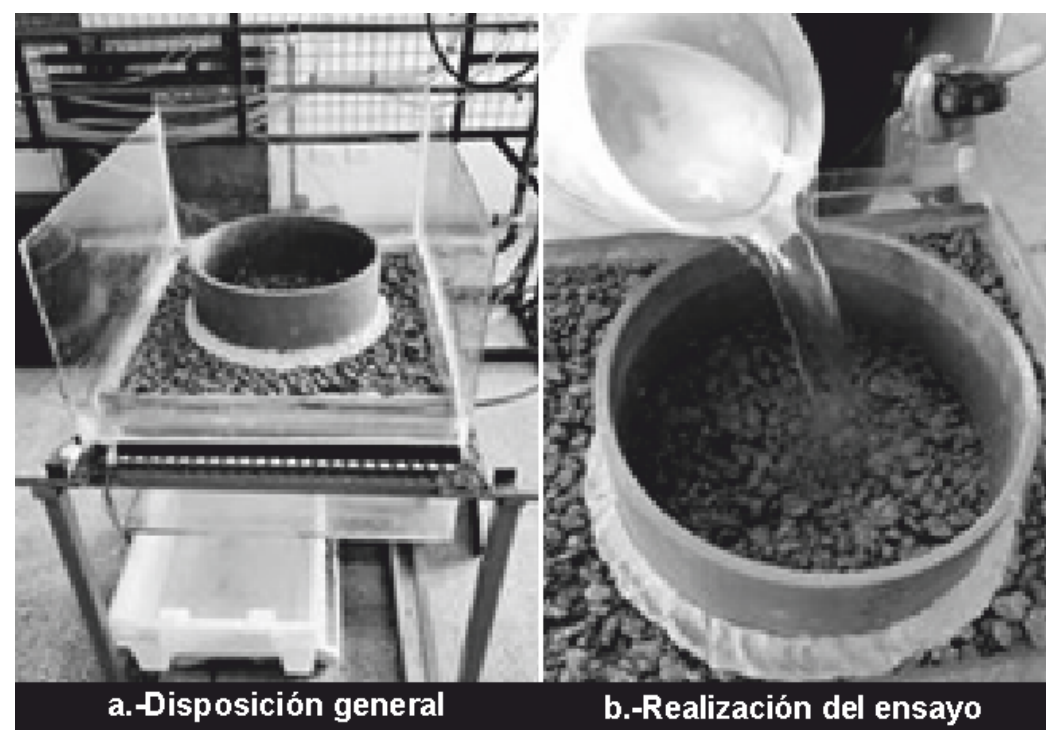

Figura 8.- Ensayo de permeabilidad ASTM C1701

La elevada permeabilidad del hormigón poroso se reduce gradualmente a medida que los pavimentos son sometidos al uso, debido a que el material fino colmata progresivamente los vacíos del material. Uno de los objetivos de los ensayos consistió en identificar la variación de las tasas de infiltración ante modificaciones del grado de colmatación de las probetas. Las muestras se colmataron incorporando escalones de arena variables entre 250 gr y 500 gr, en función de la porosidad hasta alcanzar la colmatación total. El material utilizado para colmatar las muestras de hormigón poroso es arena fina recolectada en cordones cuneta del Departamento Concordia. La arena se distribuyó homogéneamente con pincel sobre la superficie de las muestras, la cual se regó durante el tiempo necesario para forzar el ingreso al interior de las probetas. Para evitar el lavado del material fino se colocó en la base de las probetas una malla de acero inoxidable calidad AISI $304 \mathrm{~N}^{\circ} 100$ (alambre de 0,10 mm, luz de malla de 154 micrones y $36.89 \%$ de área abierta), que retiene la arena incorporada en el interior de la probeta.

\section{Simulador de lluvia}

A medida que se incrementa el grado de colmatación, las tasas de infiltración se reducen 
sustancialmente. Cuando las muestras de hormigón poroso alcanzan contenidos de arena próximos a la colmatación, las tasas de infiltración alcanzan un orden de magnitud igual o inferior al de las tormentas. Dichas tasas son variables en función de la porosidad de la muestras y de la intensidad de precipitación.

En este rango de grados de colmatación se utilizó una metodología experimental, empleando un simulador de lluvia a escala de laboratorio (Figura 9), construido a partir del diseño del Infiltrómetro Cántabro Fijo (ICF) de la Universidad de Cantabria (Sañudo F, 2014). Este simulador tiene inclinación regulable, que permite caracterizar las distintas pendientes de la superficie permeable. Cuenta con un soporte superior, constituido por una estructura metálica ubicada a $50 \mathrm{~cm}$ sobre la probeta, que se utilizó como estructura de fijación del simulador de lluvia directa, con cinco líneas de distribución, sobre las que se instalaron 15 goteros de riego por línea (75 goteros en total). La alimentación de agua al simulador se realizó a través de un tanque a nivel constante. La regulación del caudal de entrada al simulador de lluvia se realizó mediante flotámetro monoblock para pequeños caudales, marca Schillig modelo MB-60V-B, caudal máximo 40 l/h. Este dispositivo se utilizó para simular intensidades de lluvia variables entre 80 y $160 \mathrm{~mm} / \mathrm{hora}$.

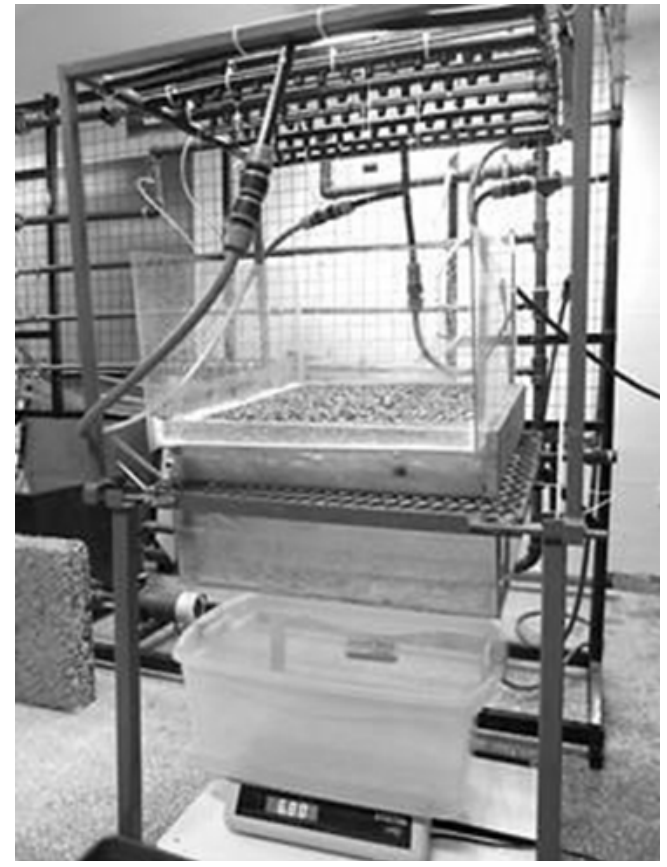

Figura 9.- Simulador de lluvia

La intensidad de precipitación se calculó de acuerdo a Ecuación (2), siendo i: intensidad en $\mathrm{mm} /$ hora, Q: caudal a la salida del flotámetro (L/hora) y A: superficie de la probeta en $\left(\mathrm{m}^{2}\right)$. Se adoptó una duración total del ensayo de 20 minutos.

$$
i=Q / A
$$


La probeta de hormigón se confinó perimetralmente mediante un marco de policarbonato, que intercepta las salpicaduras originadas por el choque de las gotas con la superficie de la probeta, reteniéndolas en el interior del volumen de control. El contacto entre ambas superficies se selló para evitar la eventual percolación lateral, que altera la hipótesis de flujo unidimensional.

El volumen que percola a través de la muestra de hormigón poroso se recolectó en un recipiente de policarbonato con cinco divisiones, ubicado en el plano inferior de la probeta. El agua proveniente del mismo descarga en un recipiente totalizador ubicado sobre una balanza electrónica, que se utilizó para registrar los pesos correspondientes a intervalos regulares de tiempo. A su vez, la baja capacidad de infiltración de las muestras colmatadas origina tasas de escorrentía variables que se registraron recolectando el escurrimiento captado en el borde más bajo de la probeta, mediante una canaleta de policarbonato, que transfiere esta fracción hacia un recipiente totalizador independiente. Para registrar los pesos correspondientes al escurrimiento superficial se utilizó también una balanza electrónica, adoptando los mismos intervalos de medición que los empleados para las tasas de infiltración. De este modo, es posible cuantificar las tasas de infiltración y escorrentía (en $\mathrm{mm} / \mathrm{h}$ y en \%) correspondientes a distintos intervalos de tiempo, vinculando estas magnitudes a la intensidad de lluvia y a la pendiente del pavimento permeable.

\section{Resultados y discusión}

Propiedades en estado fresco

La determinación del peso unitario se utilizó principalmente para corregir las sucesivas dosificaciones y para controlar parámetros en estado fresco. Todas las mezclas se ubicaron en el rango de 1700 a $1900 \mathrm{~kg} / \mathrm{m}^{3}$, encontrándose dentro de los límites para este tipo de hormigón. En el ensayo de asentamiento se verificó un asentamiento nulo o de $5 \mathrm{~cm}$ como máximo, verificándose asentamientos menores a $20 \mathrm{~mm}$, (recomendado por bibliografía para este tipo de hormigones). En todas las muestras tomadas al azar con la mano, las pastas resultaron pegajosas, lo que significa una buena adherencia pasta-agregado.

Del análisis cualitativo de la totalidad de las series, se observaron los siguientes resultados:

- Las mezclas con agregado grueso de canto rodado, presentaron una consistencia más trabajable en comparación al de basalto triturado para iguales relaciones agua/cemento.

- Se observó una mejora significativa de la cohesión de la mezcla en las series donde se utilizó aditivo superplastificante, logrando una película de recubrimiento de pasta continua y uniforme.

- Vinculado a lo anterior, al emplear el canto rodado como agregado grueso, de granulometría más completa, y sumado el efecto del aditivo plastificante, se originó un mayor contenido de pasta en la mezcla produciéndose una estructura muy cerrada, como fue el caso del pastón 4.

\section{Propiedades en estado endurecido}

\section{Resistencia a compresión}

En la primera etapa experimental se establecieron edades de ensayo de 28, 54 y 120 días para cuantificar la resistencia a la compresión. Los resultados fueron relativamente bajos, 
variando entre 5 y $18 \mathrm{MPa}$. En la Figura 10 se presentan las resistencias obtenidas en esta etapa, en función de los cuales, se rediseñaron las mezclas para la segunda etapa experimental.

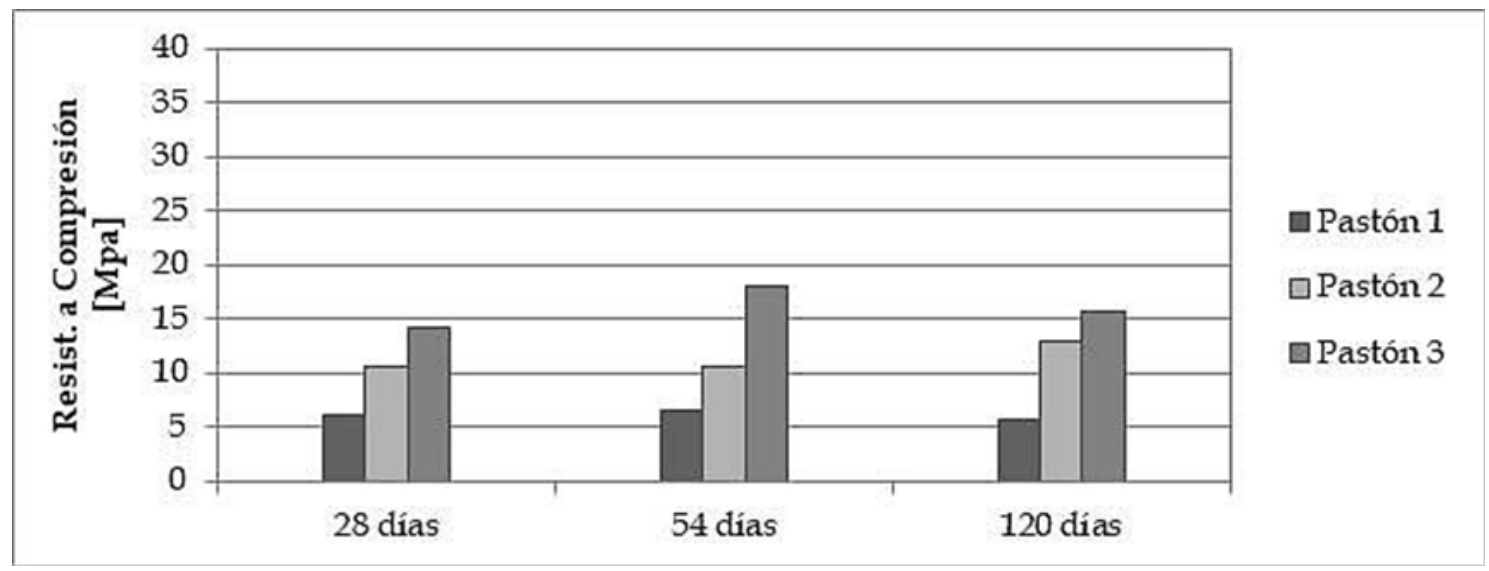

Figura 10.- Valores promedios de resistencia a compresión en MPa para los pastones 1, 2 y 3.

Los pastones 4, 5 y 6 de la segunda etapa experimental, presentaron una mayor resistencia a compresión, lográndose valores superiores a $25 \mathrm{MPa}$ para el cuarto pastón donde se obtuvo una estructura cerrada debido al empleo de canto rodado; y valores promedio superiores a $20 \mathrm{MPa}$ para los pastones 5 y 6 elaborados con basalto triturado. Durante los ensayos, realizados a los 100 días de edad, se verificó un elevado porcentaje de agregado fracturado, relacionándose este fenómeno con la forma lajosa del agregado basáltico. Esta forma angular y rugosa, así como su granulometría permiten que la trabazón sea uno de los factores preponderantes de las propiedades en estado endurecido.

En la tercera etapa experimental se lograron valores de resistencia a compresión entre 15 y $20 \mathrm{MPa}$ a los 28 días, los cuales son levemente más bajos debido al aumento de la relación a/c pero que se encuentran dentro de valores típicos. También se observó que el aumento del contenido unitario de cemento mejoró la trabajabilidad, pero no afectó en gran medida la resistencia de la estructura para el mismo grado de compactación. En la Figura 11 se presentan los resultados de resistencia a la compresión obtenidos en la segunda y tercera etapa experimental. 


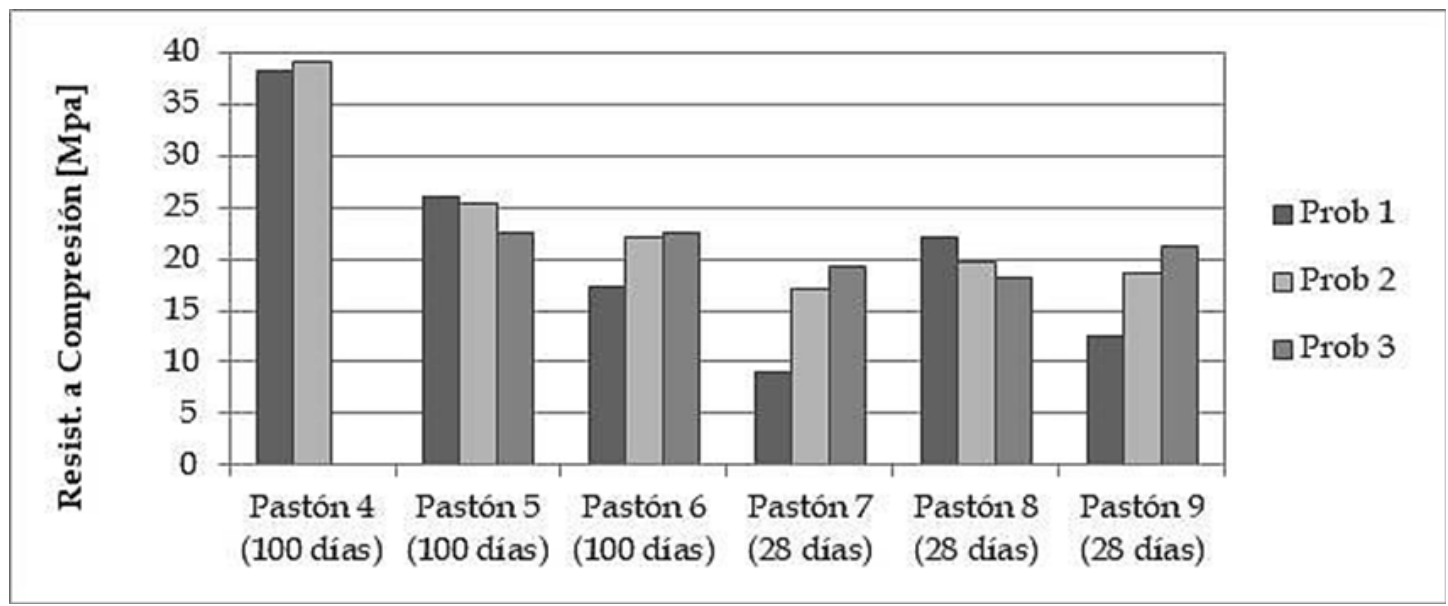

Figura 11.- Valores de resistencia a compresión en MPa para la segunda y tercera etapa experimental.

\section{Resistencia a tracción por flexión}

Para la primera etapa experimental los ensayos se realizaron a los 120 días, y los valores promedio fueron 1,40 MPa para el primer pastón, 2,35 MPa para el segundo y 2,00 MPa para el tercero. Estos valores concuerdan con lo indicado por la bibliografía, comprendidos entre 2 y $4 \mathrm{MPa}$. En la segunda etapa, debido a que se trabajaron con volúmenes de 50 litros y se elaboraron las losas para determinar las propiedades hidráulicas, no se evaluó su resistencia a flexión, lo cual sí se realizó para la tercera etapa. Los pastones 7,8 y 9 se ensayaron a la edad de 60 días y se obtuvieron valores de aproximadamente 2,5 MPa en los tres casos. En la Figura 12 se presentan los resultados de resistencia a la flexión obtenidos en la primera y tercera etapa experimental.

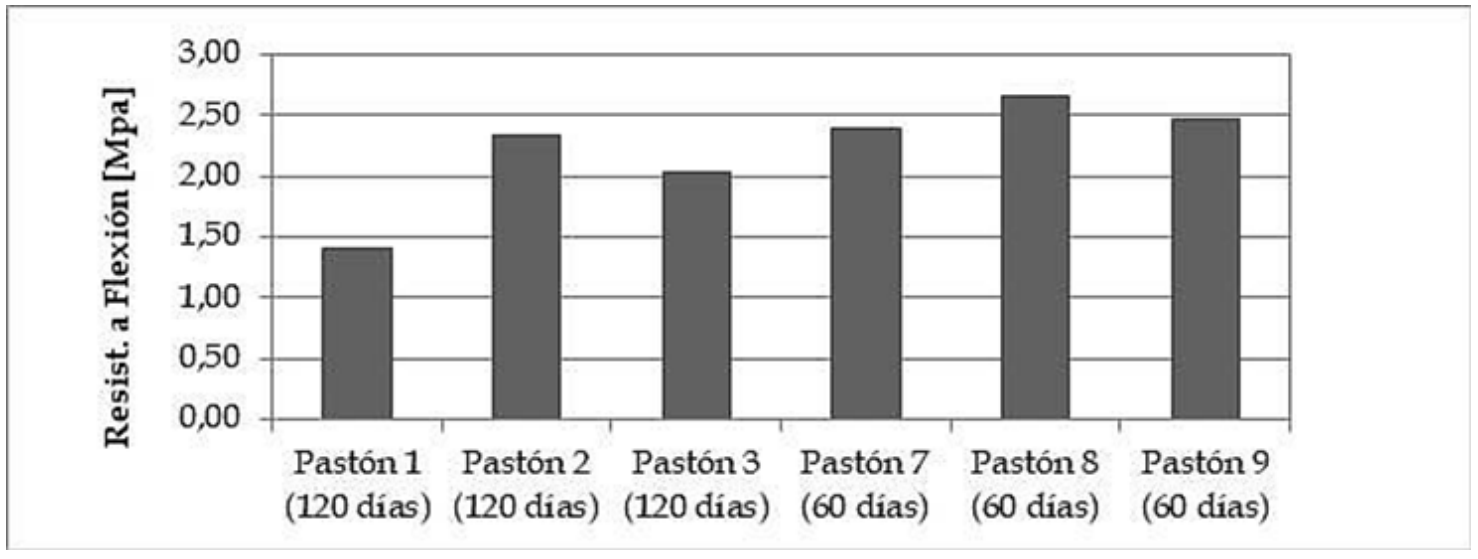

Figura 12.- Valores de resistencia a flexión en MPa para la primera y tercera etapa experimental. 


\section{Relación porosidad - resistencia de las probetas}

Del análisis de resultados de la totalidad de los pastones se observó que sólo en los pastones 4 y 5 el valor de porosidad cayó por debajo del límite inferior de $15 \%$ recomendado, y que ninguno superó el valor de $25 \%$ de porosidad, en el caso de las probetas cilíndricas. De esta manera la mayoría de las series arrojaron valores de porosidad adecuados. Los resultados de los ensayos de resistencia a la compresión, determinados para la segunda y tercera etapa se correlacionan con los valores de porosidad de las probetas en la Figura 13.

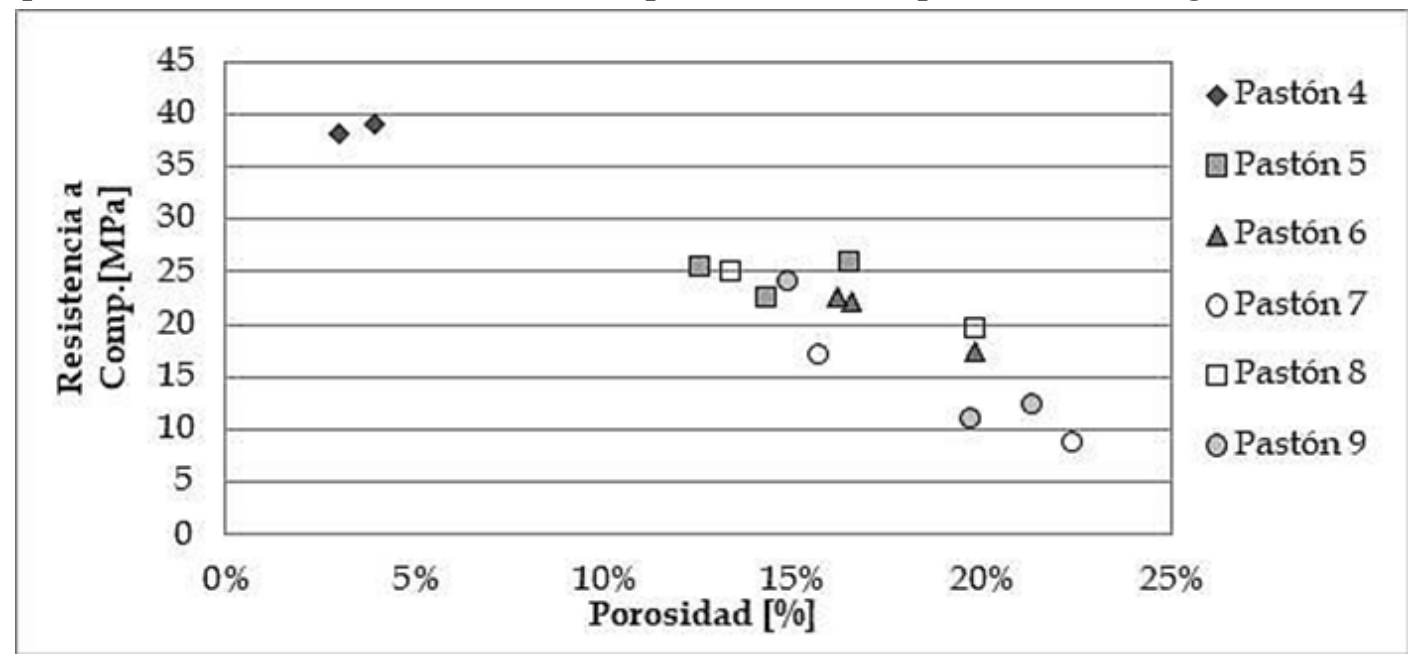

Figura 13.- Relación porosidad - resistencia a compresión para la segunda y tercera etapa experimental.

\begin{tabular}{|c|c|c|c|}
\hline Pastón & Probeta & Resistencia Compresión [Mpa] & Porosidad Total [\%] \\
4to Pastón & 1 & 38,17 & $2,99 \%$ \\
& 2 & 39,01 & $3,95 \%$ \\
5to Pastón & 1 & 25,97 & $16,44 \%$ \\
& 2 & 25,43 & $12,54 \%$ \\
& 3 & 22,58 & $14,26 \%$ \\
6to Pastón & 1 & 17,36 & $19,77 \%$ \\
& 2 & 22,2 & $16,51 \%$ \\
7mo Pastón & 3 & 22,62 & $16,18 \%$ \\
& 1 & 8,93 & $22,34 \%$ \\
8vo Pastón & 1 & 17,09 & $15,64 \%$ \\
& 2 & 25,17 & $13,33 \%$ \\
9no Pastón & 1 & 19,76 & $19,78 \%$ \\
& 2 & 12,45 & $21,29 \%$ \\
& 2 & 11,18 & $19,65 \%$ \\
\hline
\end{tabular}

Tabla 2.- Resistencia a la compresión y porosidad total de las probetas 
Las porosidades de las losas de 49x49x10 $\mathrm{cm}$ elaboradas para los ensayos de capacidad de infiltración se resumen en la Tabla 3, donde puede observarse que el pastón 7 (A) tiene una porosidad media de 25,1\%, el pastón 8 (B) de 22,9\% y el pastón 9 (C) de 12,8\%. Por ello, se considera que los rangos de porosidad característicos de cada pastón son:

Pastón A: $\eta=25 \%$

Pastón B: $\eta=20 \%$ a $25 \%$

Pastón C: $\eta=10 \%$ a $15 \%$

\begin{tabular}{|c|c|c|c|}
\hline Pastón & Losa & Porosidad Total [\%] & Porosidad Promedio [\%] \\
\hline \multirow{3}{*}{$\begin{array}{l}\text { Pastón } 7 \\
\text { (A) }\end{array}$} & A1 & 27 & \multirow{3}{*}{25,1} \\
\hline & $\mathrm{A} 2$ & 25 & \\
\hline & A3 & 24 & \\
\hline \multirow{3}{*}{$\begin{array}{l}\text { Pastón } 8 \\
\text { (B) }\end{array}$} & B1 & 23 & \multirow{3}{*}{22,9} \\
\hline & B2 & 24 & \\
\hline & B3 & 22 & \\
\hline \multirow{3}{*}{$\begin{array}{l}\text { Pastón } 9 \\
\text { (C) }\end{array}$} & C1 & 9 & \multirow{3}{*}{12,8} \\
\hline & $\mathrm{C2}$ & 14 & \\
\hline & C3 & 16 & \\
\hline
\end{tabular}

Tabla 3.- Porosidad de las losas para cada pastón de la tercera etapa experimental.

\section{Ensayos de Permeabilidad}

\section{Pastón A $(\eta=25 \%)$}

El pastón A es el de mayor porosidad de las mezclas estudiadas: en promedio, su porosidad es de 25,13\%, por ello se ha considerado que los resultados caracterizan el comportamiento de muestras del $25 \%$ de porosidad. El peso de arena necesario para colmatar completamente la muestra varía entre 9,75 y $10,50 \mathrm{~kg}$, que equivalen a cargas de sedimento variables entre $39 \mathrm{~kg} / \mathrm{m}^{2}$ y $42 \mathrm{~kg} / \mathrm{m}^{2}$.

Cada escalón de carga se expresó como porcentaje del máximo peso de arena que admiten las muestras, con el objeto de generar curvas relativas a la colmatación máxima. La representación gráfica de la variación de las tasas de infiltración (I en $\mathrm{mm} / \mathrm{h}$ ) en función del grado de colmatación de las muestras (en \%) se presenta en la Figura 14. Las tasas máximas de infiltración correspondientes a probetas limpias (Io en $\mathrm{mm} / \mathrm{h}$ ) tienen un orden de magnitud que varía entre $40.000 \mathrm{~mm} / \mathrm{h}$ y $100.000 \mathrm{~mm} / \mathrm{h}$.

A medida que se incrementa el contenido de arena incorporado a las muestras se puede observar la drástica reducción de la capacidad de infiltración, hasta valores de un orden de magnitud comprendido entre 350 y $450 \mathrm{~mm} / \mathrm{h}$ correspondientes al último ensayo con permeámetro ASTM C1701. 
La capacidad de infiltración correspondiente a un grado de colmatación del 100\% se denominó Ic (Infiltración de muestras colmatadas o Infiltración final). Su valor se obtuvo a partir de los ensayos con simulador de lluvia para pendiente horizontal e intensidades variables entre 100 y $140 \mathrm{~mm} / \mathrm{h}$. Para las tres muestras ensayadas Ic varía entre 50 y $86 \mathrm{~mm} / \mathrm{h}$, adoptándose un valor de Ic (en $\mathrm{mm} / \mathrm{h}$ ) equivalente al promedio de los valores obtenidos para las muestras. La relación Ic/Io (razón entre la tasa de infiltración final y la tasa correspondiente a probetas limpias) indicada varía entre $0,05 \%$ y $0,20 \%$. Es decir, en todos los casos la colmatación total de las muestras genera una reducción superior al $99 \%$ con respecto a la capacidad de infiltración de muestras limpias.

\section{Pastón B ( $\eta=20 \%$ a 25\%)}

La porosidad media del pastón B es de $22,85 \%$, por lo cual lo cual se lo ha adoptado para caracterizar mezclas de porosidades variables entre $20 \%$ y $25 \%$. La carga de sedimento requerida para la colmatación total de las muestras varió entre $36 \mathrm{~kg} / \mathrm{m}^{2}$ y 46 kg/m².

En la Figura 15 se presentan los resultados de los ensayos de infiltración, donde puede observarse que el orden de magnitud de las tasas iniciales de infiltración (Io en $\mathrm{mm} / \mathrm{h}$ ) varía en un rango entre $17.000 \mathrm{~mm} / \mathrm{h}$ y $38.000 \mathrm{~mm} / \mathrm{h}$. La tasa de infiltración final de las probetas completamente colmatadas (Ic en $\mathrm{mm} / \mathrm{h}$ ) varía entre 23 y $51 \mathrm{~mm} / \mathrm{h}$.

La relación entre las tasas de infiltración de probetas colmatadas vs probetas limpias (Ic/ Io) varía entre $0,10 \%$ y $0,21 \%$. En todos los casos la colmatación de las muestras genera reducciones superiores al 99 \% de la capacidad de infiltración de muestras limpias.

\section{Pastón C ( $\eta=15 \%)$}

La porosidad media del pastón C es de $12,75 \%$, por lo cual lo cual se lo ha adoptado para caracterizar mezclas de porosidades de hasta $15 \%$.

En Figura 16 se presentan los resultados de los ensayos. El orden de magnitud de las tasas iniciales de infiltración (Io en $\mathrm{mm} / \mathrm{h}$ ) varía en un rango entre $6.000 \mathrm{~mm} / \mathrm{h}$ y $12.000 \mathrm{~mm} / \mathrm{h}$. La tasa de infiltración final de las probetas completamente colmatadas (Ic en $\mathrm{mm} / \mathrm{h}$ ) varía entre 19 y $36 \mathrm{~mm} / \mathrm{h}$. Dichos valores se obtuvieron para cargas de sedimento variables entre $14 \mathrm{~kg} / \mathrm{m}^{2}$ y $27 \mathrm{~kg} / \mathrm{m}^{2}$.

La relación entre las tasas de infiltración de probetas colmatadas vs probetas limpias (Ic/ Io) varía entre $0,15 \%$ y $0,57 \%$. En todos los casos la colmatación de las muestras genera reducciones superiores al $99 \%$ de la capacidad de infiltración de muestras limpias.

\section{Curvas de ajuste de la capacidad de infiltración de los pastones}

Para caracterizar la variación de la capacidad de infiltración en función del grado de colmatación de cada pastón se ajustaron los valores experimentales a diferentes curvas, seleccionando en cada caso la curva de ajuste de mayor coeficiente de correlación.

En el caso del pastón A se seleccionó una función cuadrática, para la cual se obtuvo un 
coeficiente de correlación $r=0,9822$. El pastón $B$ se ajustó mediante una función exponencial $(\mathrm{r}=0.9809)$ y el pastón $\mathrm{C}$ a un modelo logístico $(\mathrm{r}=0.7158)$.

En la Figura 17 se comparan las curvas de ajuste de los tres pastones, cuyos valores se resumen en la Tabla 4.

Comparando las tasas de infiltración de las muestras limpias (Io en $\mathrm{mm} / \mathrm{h}$ ) puede observarse que su orden de magnitud varía entre $7000 \mathrm{~mm} / \mathrm{h}$ y $44000 \mathrm{~mm} / \mathrm{h}(0,19 \mathrm{~cm} / \mathrm{s}$ a $1,22 \mathrm{~cm} / \mathrm{s})$ para el rango de porosidades analizadas. El orden de magnitud de estos resultados concuerda con los valores informados por la bibliografía, entre otros por (Kevern et al., 2008) y (Iowa State University, 2016).

Las tasas del Pastón A son, en orden de magnitud, 6 veces superiores a las del pastón C. Mientras que la capacidad de infiltración del pastón B las triplican. La capacidad de infiltración decrece rápidamente a medida que se incrementa el grado de colmatación, hasta alcanzar tasas variables entre $30 \mathrm{~mm} / \mathrm{h}$ y $60 \mathrm{~mm} / \mathrm{h}$.

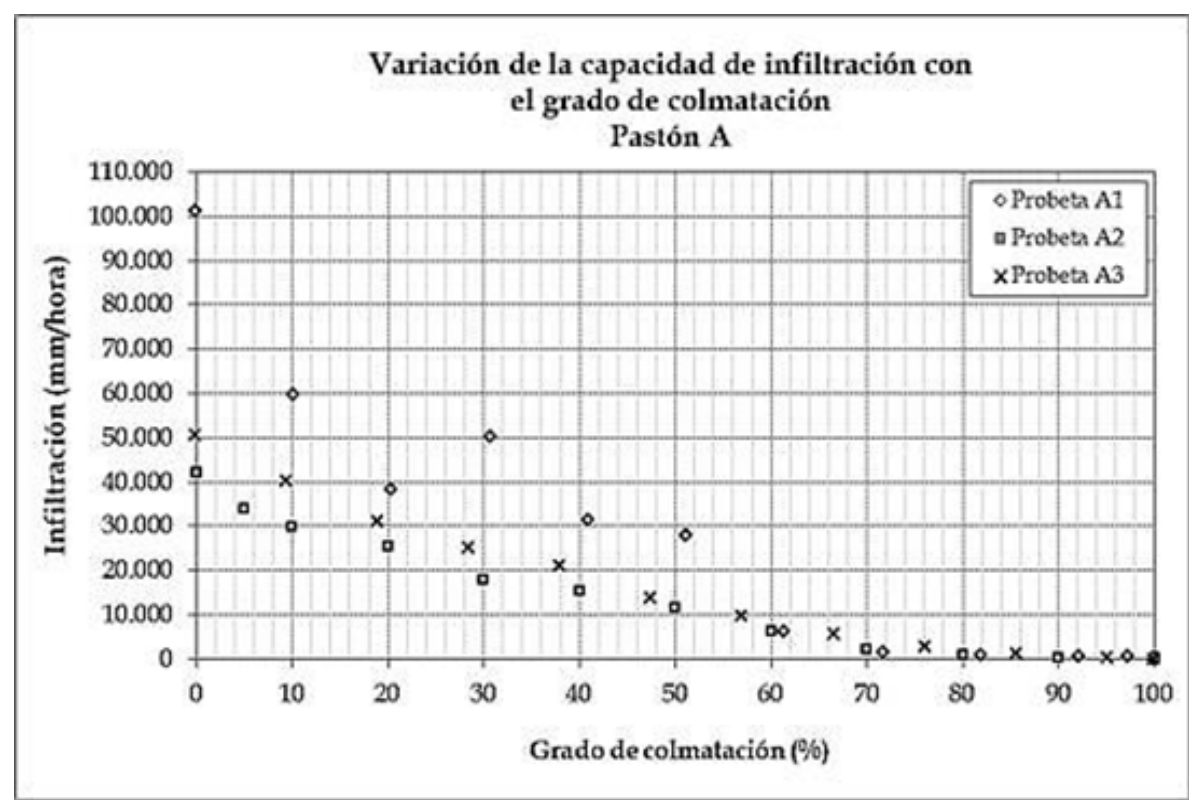

Figura 14.- Variación de la capacidad de infiltración con el grado de colmatación - Pastón A. 


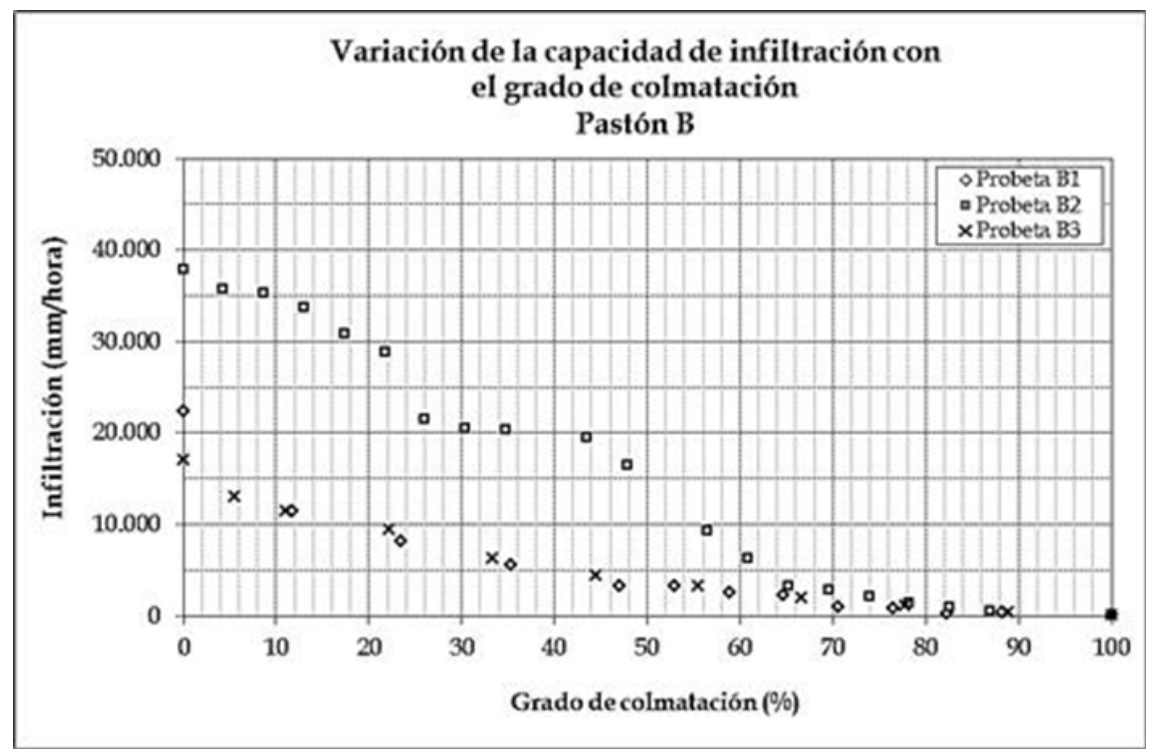

Figura 15.- Variación de la capacidad de infiltración con el grado de colmatación - Pastón B.

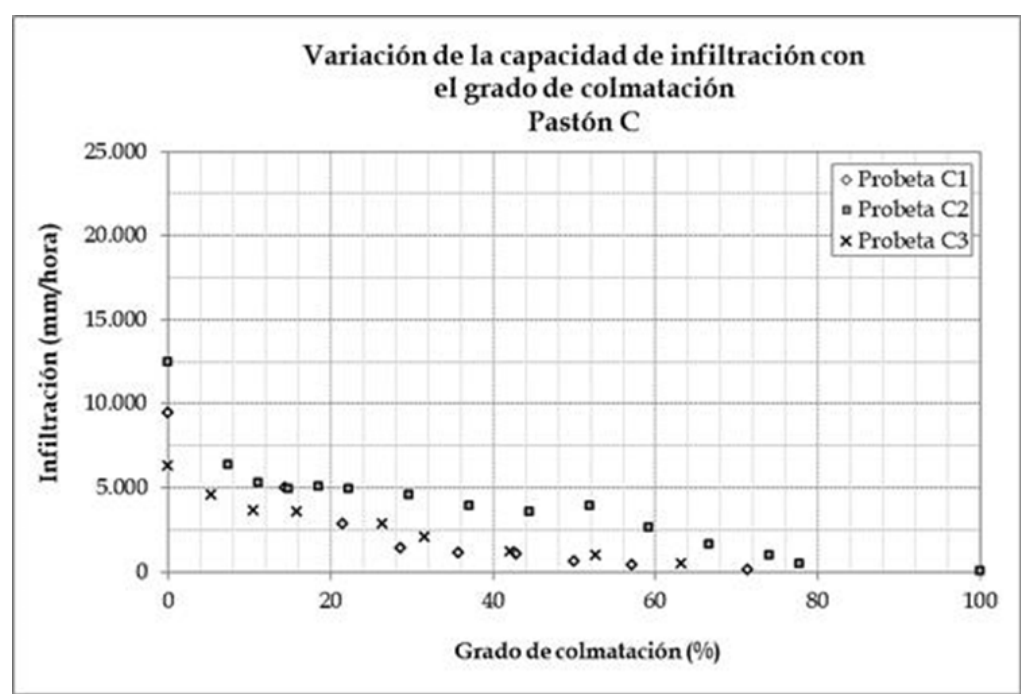

Figura 16.- Variación de la capacidad de infiltración con el grado de colmatación - Pastón C. 


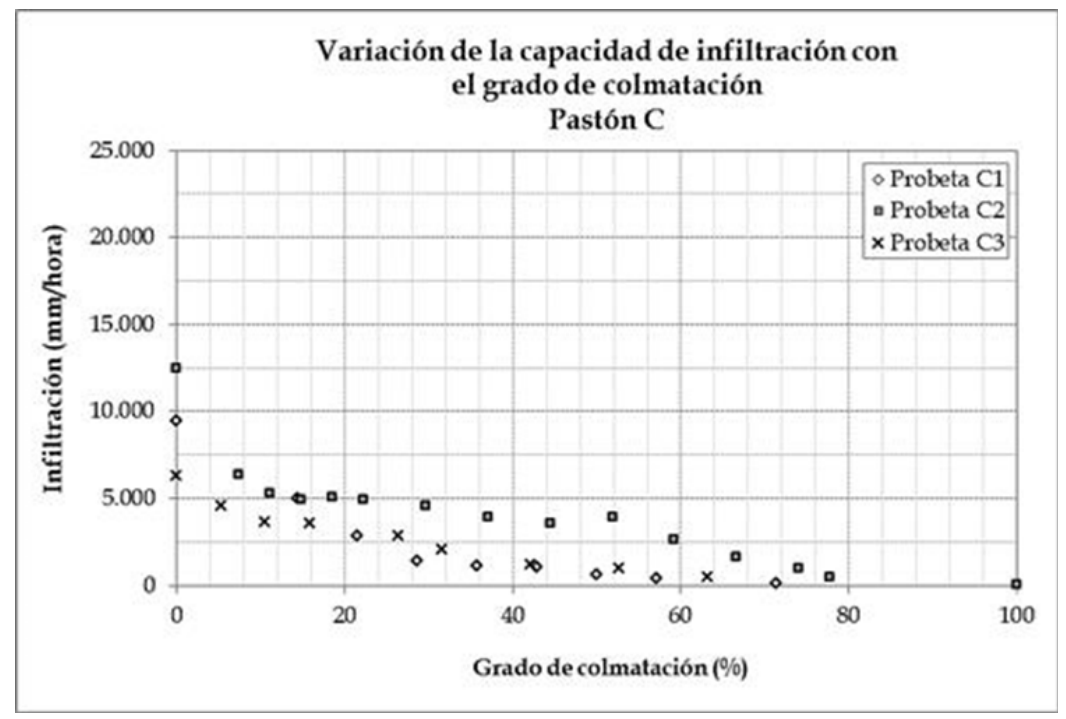

Figura 17.- Comparación de las curvas de ajuste

\begin{tabular}{|c|c|c|c|}
\hline Grado de colmatación (\%) & $\begin{array}{c}\text { Pastón A } \\
(\eta=25 \%)\end{array}$ & $\begin{array}{c}\text { Pastón B } \\
(\eta=20 \% \text { a } 25 \%)\end{array}$ & $\begin{array}{c}\text { Pastón C } \\
(\eta=15 \%)\end{array}$ \\
0 & 43.659 & 18.606 & 6.903 \\
5 & 39.461 & 15.580 & 5.926 \\
10 & 35.475 & 13.046 & 5.104 \\
15 & 31.700 & 10.924 & 4.408 \\
20 & 28.137 & 9.147 & 3.816 \\
25 & 24.785 & 7.659 & 3.310 \\
30 & 21.645 & 6.413 & 2.877 \\
35 & 18.716 & 5.370 & 2.503 \\
40 & 15.999 & 4.497 & 2.181 \\
45 & 13.493 & 3.765 & 1.903 \\
50 & 11.199 & 3.153 & 1.662 \\
55 & 9.116 & 2.640 & 1.452 \\
60 & 7.245 & 2.211 & 1.270 \\
65 & 5.585 & 1.851 & 1.111 \\
70 & 4.137 & 1.550 & 973 \\
75 & 2.900 & 1.298 & 852 \\
80 & 1.875 & 1.001 & 658 \\
85 & 1.353 & 751 & 494 \\
90 & 902 & 501 & 329 \\
95 & 451 & 250 & 165 \\
100 & 72 & 46,5 & 30 \\
\hline & & & \\
\hline
\end{tabular}

Tabla 4.- Tasas de infiltración ajustadas de los tres pastones $(\mathrm{mm} / \mathrm{h})$ 


\section{Relación porosidad - resistencia - tasa de infiltración de muestras limpias}

En base a los resultados de los ensayos realizados sobre muestras limpias, es posible analizar la siguiente la relación empírica que vincula las tres variables analizadas: resistencia a la compresión y tasas de infiltración de las muestras limpias vs porosidad para pavimentos de hormigón poroso construidos con áridos del departamento Concordia, Provincia de Entre Ríos.

La misma se presenta en la Figura 18, donde se observa que a medida que se incrementa la porosidad de la muestra la resistencia a la compresión se reduce con una tendencia marcadamente lineal (coeficiente de determinación $\mathrm{R}^{2}=0,89$ )

En el rango de porosidades analizado (10\% a 25\%), las resistencias a compresión correspondientes a la recta de ajuste se ubican en el rango $7 \mathrm{Mpa}$ a $30 \mathrm{Mpa}$, los cuales se encuentran dentro de valores típicos informados por la bibliografía.

A su vez, la permeabilidad aumenta drásticamente ante incrementos de la porosidad. Una posible curva de ajuste a los resultados experimentales se indica en línea de trazos, que corresponde a un modelo de tipo logístico. Sin embargo, se considera que es necesaria la realización de mayor cantidad de ensayos para su definición.

Las tasas de infiltración comprenden el rango entre $6300 \mathrm{~mm} / \mathrm{h}(0,18 \mathrm{~cm} / \mathrm{s})$ y 102.000 $\mathrm{mm} / \mathrm{h}(2,83 \mathrm{~cm} / \mathrm{s})$, los cuales también se ubican en el rango de valores típicos de este tipo de material.

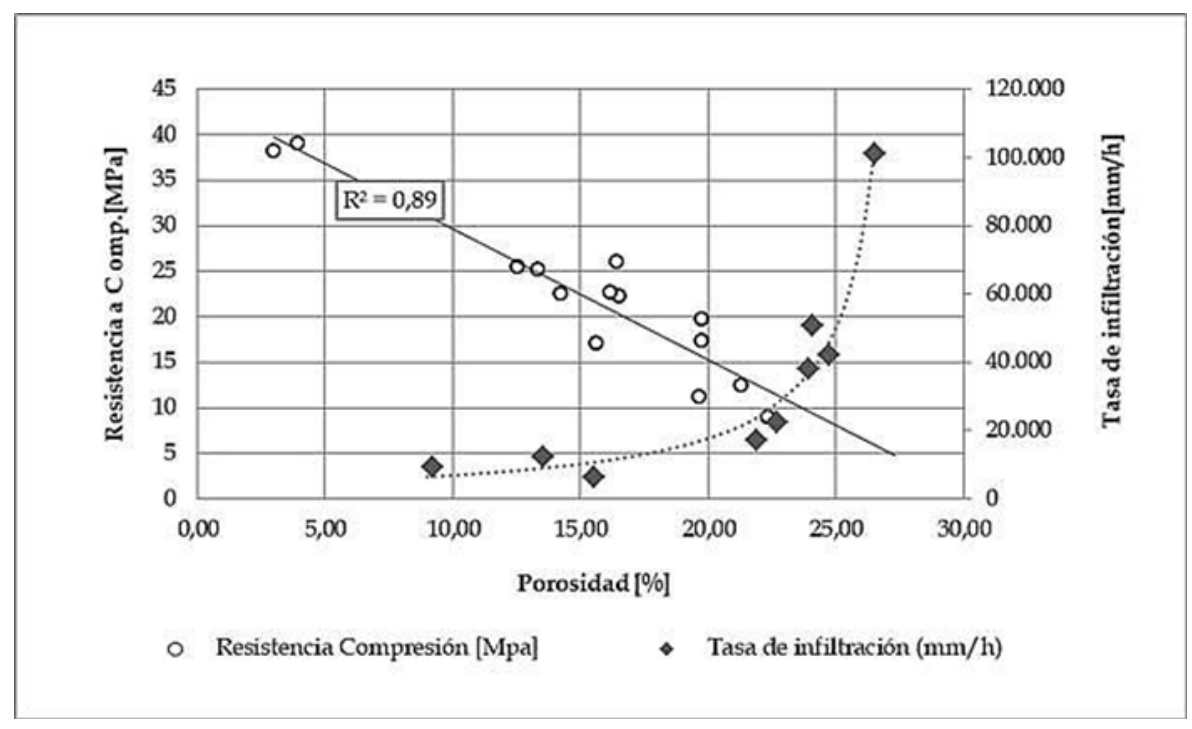

Figura 18.- Relación porosidad - resistencia - tasa de infiltración 


\section{Conclusiones}

Los resultados de este trabajo permitieron caracterizar el comportamiento estructural e hidrológico de distintas mezclas de hormigón poroso construidas con áridos de la Provincia de Entre Ríos, verificando las correlaciones entre la porosidad y la resistencia a la compresión, flexión y densidad de la mezcla, los cuales arrojaron valores acordes a los recomendados por la bibliografía consultada.

Para muestras elaboradas con basalto triturado, al incrementarse la porosidad entre $15 \%$ y 22\% la resistencia a compresión se modificó entre $25 \mathrm{MPa}$ y $10 \mathrm{MPa}$. La resistencia a tracción por flexión promedio arrojó valores de 2,50 $\mathrm{MPa}$. El peso unitario de todas las mezclas se ubicó en el rango de 1700 a $1900 \mathrm{~kg} / \mathrm{m}^{3}$, encontrándose dentro de los límites para este tipo de hormigón. La relación agua/cemento de las mezclas se modificó entre 0,25 y 0,30, observándose una mejora significativa de la cohesión de la mezcla con la incorporación de aditivo superplastificante.

Para cuantificar la variación de la capacidad de infiltración ante grados crecientes de colmatación se realizaron 131 ensayos de permeabilidad (Norma ASTM C1701) sobre muestras de tres rangos diferentes de porosidad.

La capacidad de infiltración máxima correspondiente a probetas limpias (Io en $\mathrm{mm} / \mathrm{h}$ ) varía entre $7000 \mathrm{~mm} / \mathrm{h}$ y $44000 \mathrm{~mm} / \mathrm{h}(0,19 \mathrm{~cm} / \mathrm{s}$ a $1,22 \mathrm{~cm} / \mathrm{s})$ para el rango de porosidades analizadas. Incluso para grados de colmatación muy altos (entre $70 \%$ y $80 \%$ de la colmatación total), estos pavimentos presentan tasas de infiltración muy elevadas, sin generar escurrimiento superficial.

En las muestras más permeables (porosidad del orden del 25\%), las tasas de infiltración varían entre Io $=44.000 \mathrm{~mm} / \mathrm{h}(1,22 \mathrm{~cm} / \mathrm{s})$ en muestras limpias hasta $\mathrm{Ic}=70 \mathrm{~mm} / \mathrm{h}$ (muestras completamente colmatadas (Ic). La carga de sedimento requerida para la colmatación total alcanzó un promedio de $40 \mathrm{~kg} / \mathrm{m}^{2}$.

En el caso de muestras de porosidad intermedia (20\% a 25\%), la magnitud de la tasa de infiltración varía entre $18.000 \mathrm{~mm} / \mathrm{h}(0,50 \mathrm{~cm} / \mathrm{s})$ para muestras limpias y $45 \mathrm{~mm} / \mathrm{h}$ para muestras completamente colmatadas. La carga de sedimento requerida para la colmatación total alcanzó los $36 \mathrm{~kg} / \mathrm{m} 2$.

En las muestras de menor porosidad (10 a 15\%) la permeabilidad varía entre Io=7000 $\mathrm{mm} / \mathrm{h}(0,19 \mathrm{~cm} / \mathrm{s})$ e Ic=30 $\mathrm{mm} / \mathrm{h}$ correspondiente a un grado de colmatación total, que se alcanzó con cargas de sedimento variables entre $14 \mathrm{~kg} / \mathrm{m}^{2}$ y $27 \mathrm{~kg} / \mathrm{m}^{2}$.

Comparando los resultados obtenidos en todos los ensayos, el progreso completo del fenómeno de colmatación del pavimento poroso por aportes de sedimentos genera tasas finales de infiltración iguales o inferiores al 1\% del valor inicial correspondiente a muestras limpias. De allí, la importancia de adecuada limpieza y mantenimiento periódicos de este tipo de material. 
La serie de ensayos realizada permitió identificar algunos factores determinantes de las propiedades de estos hormigones especiales. El propósito de los autores es continuar con los ajustes de dosificación y elaboración empleando nuevos agregados, con el fin de desarrollar un método de dosificación que considere el grado de compactación de la mezcla. Estos resultados constituyen un punto de partida para el conocimiento y utilización de este material en nuestra región (Mesopotamia argentina), ampliando la base de conocimientos para la caracterización de los parámetros mecánicos e hidrológicos de este material.

\section{Agradecimientos}

A los becarios alumnos de investigación de la Facultad Regional Concordia de la UTN: Sergio Gonfiotti, Vanesa Cayecul, María Belén Rodriguez y Eugenio Sauré.

A los becarios graduados: Ing. Martín Wendler, Ing. Gerardo Sasso e Ing. Elisa Fracaro.

A la empresa constructora COINAR SRL de la ciudad de Concordia. 


\section{Referencias}

ASTM, 2009. "ASTM C1701 / C1701M-09. Standard Test Method for Infiltration Rate of In Place Pervious Concrete". West Conshohocken, PA: www.astm.org.

GIHHA, 2014. "Criterios de Planeamiento y Herramientas de Diseño de Sistemas de Drenaje Urbano sustentables para la provincia de Entre Ríos". Concordia: Grupo de Investigación en Hidrología e Hidráulica Aplicada (GIHHA). Universidad Tecnológica Nacional.

Hernández, C., Collura, A. \& Mansilla, G., 2008. “Hormigón poroso”. Centro Técnico Loma Negra.

Iowa State University, 2016. "Pervious Concrete Physical Characteristics and Effectiveness in Stormwater Pollution Reduction". Ames, Iowa,USA: Institute for Transportation - Iowa State University.

Kevern, J.T., Schaefer, V.R., Wang, K. \& Suleiman, M.T., 2008. "Pervious Concrete Mixture Proportions for Improved Freeze-Thaw Durability". West Conshohocken, PA, USA: Journal of ASTM International, Vol. 5, No. 2.

Sañudo F, L.A., 2014. "Análisis de la infiltración de agua de lluvia en firmes permeables con superficies de adoquines y aglomerados porosos para el control en origen de inundaciones". Santander: Universidad de Cantabria. Escuela Técnica Superior de Ingenieros de Caminos, Canales y Puertos. 